\title{
Composite phenomenology as a target for lattice QCD
}

\section{Thomas DeGrand*}

Department of Physics, University of Colorado, Boulder, CO 80309, USA

E-mail: thomas.degrandecolorado.edu

\section{Ethan T. Neil}

Department of Physics, University of Colorado, Boulder, CO 80309, USA

RIKEN-BNL Research Center, Brookhaven National Laboratory,

Upton, New York 11973, USA

E-mail: ethan.neil@colorado.edu

\begin{abstract}
Some recent beyond Standard Model phenomenology is based on new strongly interacting dynamics of $S U(N)$ gauge fields coupled to various numbers of fermions. When $N=3$ these systems are analogues of QCD, although the fermion masses are typically different from - and heavier than - the ones of real world QCD. Many quantities needed for phenomenology from these models have been computed on the lattice. We are writing a guide for these phenomenologists, telling them about lattice results. We'll tell you (some of) what they are interested in knowing.
\end{abstract}

The 36th Annual International Symposium on Lattice Field Theory - LATTICE2018

22-28 July, 2018

Michigan State University, East Lansing, Michigan, USA.

\footnotetext{
* Speaker.
} 
Phenomenologists continue to construct and analyze a wide variety of theories for physics beyond the Standard Model (BSM). Some of these theories contain nonperturbative systems - nonAbelian gauge fields coupled to fermions (and perhaps scalars) in which the gauge symmetry is unbroken and the gauge dynamics are confining. Many of these systems are accessible to lattice simulation. A subset of them are so accessible to lattice simulation that they have (probably) already been simulated - the gauge group is $S U(3)$ and the matter content is a set of fundamental representation fermions. What makes them different from real world QCD is that generally the pseudoscalar to vector meson mass ratio is bigger than in Nature. The corresponding lattice data for QCD at unphysically heavy quark masses exists, but it is generally thought of merely an intermediate result on the way to the QCD physical point. This means that although such lattice results could have an impact in BSM phenomenology, they are (by and large) not presented in a way which is accessible to researchers outside the lattice community for such purposes.

We are trying to collect lattice data which might impact beyond-Standard-Model phenomenology and present it to the community working in that area [1]. You lattice people are not really the audience for the paper we want to write. However, it's your data we are trying to collect. We may not have found the best examples of the things we want to show. The purpose of this writeup is to tell you, a lattice QCD practitioner, what selected phenomenologists have told us they are interested in, and to show you some of what we have found.

Our focus is on BSM models including a non-perturbative sector that resembles "heavy QCD": $\mathrm{SU}(3)$ with fundamental-irrep fermions heavier relative to the confinement scale than the light quarks of real-world QCD. Generically, these systems are examples of "hidden valleys" - new confining sectors with some weak coupling to the visible sector of the Standard Model. Early representative examples include Refs. [2, 3, 4, 5]. Some constructions are pointed at the hierarchy problem; some at dark matter. Quantities which appear most often in them, which might be lattice targets are:

- spectroscopy (of course) - but some translation of scales from QCD is required,

- the pseudoscalar decay constant and other parameters of the low energy chiral effective theories (most useful for extracting Higgs properties from the low energy effective field theory),

- decay constants, representing the matrix element of some strong bound state to "vacuum"; these are necessary to describe the decay of bound states through other interactions, e. g. a Z-boson or a dark photon. Certain decay constants, such as the vector (and perhaps axial vector), also appear in phenomenological descriptions such as vector meson dominance which are prevalent in the strongly-coupled BSM literature.

- other simple matrix elements, for example matrix elements of the scalar current, which determine the Higgs boson coupling to the new physics sector. An example of this coupling is the nuclear sigma term (which describes the coupling of the Higgs to the nucleon in a direct detection dark matter decay amplitude). If dark matter is composite there is the analog matrix element coupling the Higgs to some scalar dark current.

These are very general lists, encompassing much of what is done in lattice QCD. Of course, that is the point; most existing lattice QCD calculations, particularly at heavy fermion mass, already 
contain results which can be of interest for BSM phenomenology - so long as they are presented in the right way! We now move on to two more concrete examples and discuss where lattice results could be most impactful, beginning with "twin Higgs".

Little beyond-Standard-Model phenomenology directed at solving the hierarchy problem involves QCD-like dynamics. One exception is the twin Higgs model which introduces a copy of every Standard Model fermion, interacting with a new strong $S U(3)$ gauge symmetry with a different confinement scale. The original reference is [6] and recent papers are [5, 7, 8]. $S U(3)$ is mandatory for graph cancellation in loops between Standard Model particles and their twins. Otherwise, there are many variant models.

The original reference [6] has a copy of every Standard Model fermion. The twin fermion masses are different than the Standard Model ones because the Yukawa couplings are different. Obviously, properties of the strongly interacting twin sector are those of full QCD, but with different fermion masses. Later papers remind us that having many more light particles than are already in the Standard Model is bad for nucleosynthesis, so the full twin scenario seems to be disfavored (unless the scale of the twin sector is very high, or unless all the light constituents can decay to Standard Model particles before nucleosynthesis).

The next set of models restrict the twin quarks only to be partners of the top and bottom quarks. The justification for doing this is that the top quark has the biggest Yukawa coupling and is the biggest player in the hierarchy problem, the quadratic dependence of the Higgs mass on higher new physics scales. It is reminiscent of partial compositeness, where the quarks get their mass by mixing with some composite operator. Most of the phenomenology of this scenario restricts itself to the top and bottom quark doublet.

Several groups, including Refs. $[5,7]$ have written about this scenario. With $b$ quarks heavier than the scale of glueball bound states, the spectroscopy is very different from real world QCD: there are glueballs, which are basically quenched glueballs, and there are $\bar{b} b$ quarkonia, basically quenched quarkonia. (The mirror top quarks decay as in the real world.) The quarkonia can only decay by glueball emission.

The lattice literature on quarkonia does not seem to have affected twin - related phenomenology. We could not find any lattice spectroscopy away from the physical $c$ and $b$ masses. This would be interesting (and trivial to do, if you have the code). Phenomenologists know about quenched lattice glueball spectroscopy and cite Refs. $[9,10]$. They care about the coupling of glueballs to quarkonia. The one lattice paper on this we know is Ref. [11]. A quick glance does not reveal many citations to this by modern BSM phenomenologists. It's an old paper - can one do better?

Our second example comes from dark matter. At present there are more lattice targets in dark matter phenomenology. Ref. [12] is a survey of confining systems which have a place there.

Self interacting dark matter is characterized by having a dominant decay process $3 \rightarrow 2$. Some phenomenology assumes that the dark matter is bound states. In $[13,14]$ the dark matter is the pions of a hidden sector. A recent proposal is [15]. It is an explicit model with $S U(3)$ gauge dynamics with $N_{f}=3$ light flavors, but (unlike QCD) it has $m_{P S} / m_{V} \sim 1 / 2$. The authors are interested in computing the $3 \rightarrow 2$ amplitude in this system. They are also interested in properties of their vector meson. When a "dark photon" is included in the dark sector, it mixes not only with the ordinary photon via a term in the Lagrangian $\left(\mathscr{L}_{I} \sim \varepsilon B_{\mu \nu} F_{\mu \nu}\right)$, but also with the equivalent 
of the rho meson. This mixing involves the vector meson decay constant ( $f_{V}$ in QCD language), which, to set conventions, we define as $\left\langle 0\left|\bar{u} \gamma_{i} d\right| V\right\rangle=m_{V}^{2} f_{V} \varepsilon_{i}$.

Workers in this genre compute these quantities using a phenomenological effective Lagrangian, basically the usual chiral Lagrangian for the Goldstones augmented by extra vector meson fields.

$$
\mathscr{L}=\frac{F^{2}}{4} \operatorname{Tr}\left(D_{\mu} U D^{\mu} U^{\dagger}\right)-\frac{1}{8} \operatorname{Tr} G_{\mu v} G^{\mu v}+\ldots
$$

which is built of the Goldstone field $U=\exp (i \Phi / F)$ and vector mesons $V_{\mu}$ introduced via covariant derivative

$$
D_{\mu} \Phi=\partial_{\mu} \Phi+\frac{i g}{2}\left[\Phi, V_{\mu}\right]
$$

They have a self coupling from $G_{\mu v}=\partial_{\mu} V_{v}-\partial_{\nu} V_{\mu}$. The $\ldots$ in $\mathscr{L}$ includes phenomenological $V$ mass terms, couplings, and so on. The acronym "KSRF" (Kawarabayashi, Suzuki, Riadzuddin, Fayazuddin $[16,17])$ labels results from these models. Ref. [18] is a survey of them.

Phenomenologists get their $3 \rightarrow 2$ vertices out of a combination of the Wess-Zumino-Witten term in a chiral Lagrangian, and the coupling of a vector meson to pseudoscalars, $g_{V P P}$. They infer $f_{V}$ and $g_{\rho \pi \pi}$ from the KSRF relations,

$$
f_{V}=\sqrt{2} \frac{f_{P S}}{M_{V}}
$$

and

$$
g_{V P P}=\frac{M_{V}}{f_{P S}}
$$

In these conventions the vector meson decay width is

$$
\Gamma(V \rightarrow P P) \simeq \frac{g_{V P P}^{2}}{48 \pi m_{V}^{2}}\left(m_{V}^{2}-4 m_{P S}^{2}\right)^{3 / 2}
$$

and

$$
\Gamma\left(V \rightarrow e^{+} e^{-}\right)=\frac{4 \pi \alpha^{2}}{3} m_{V} f_{V}^{2}\langle q\rangle^{2}
$$

where $\langle q\rangle$ is the average quark charge in the valence wave function.

Does this phenomenology produce reasonable results? The situation for $f_{V}$ is shown in panel (a) of Fig. 1, showing direct lattice results, KSRF predictions from lattice data, and experimental results (from radiative decays of vector mesons), and the KSRF relation from the real world $m_{\rho}$ and $f_{P S}$. Yes, phenomenology works.

There are now many direct lattice calculations of $g_{V P P}$ from simulations in finite volume a la Lüscher. Lattice data from several groups is displayed in panel (b) of Fig. 1, along with the KSRF relation itself, evaluated using the physical values of $m_{V}$ and $f_{P S}$. The agreement of lattice data with the relation is again excellent.

Finally, we briefly discuss scale setting. Lattice calculations produce only dimensionless ratios; some physical quantity must be chosen to remove the lattice spacing dependence from these ratios and present final results in physical units like $\mathrm{GeV}$. The same procedure is needed to use lattice QCD results in the context of BSM phenomenology, but in this context the physical units will be different, and often variable over a wide range depending on the model parameters. To 

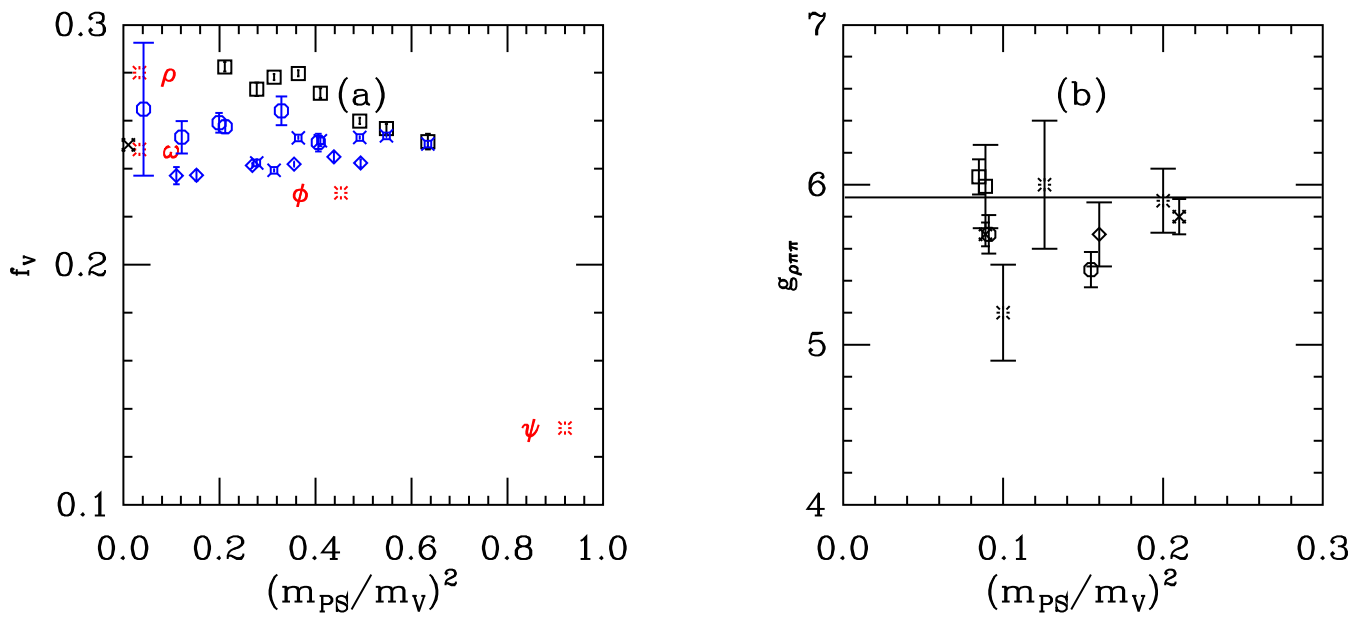

Figure 1: KSRF physics from the lattice: (a) Vector meson decay constant $f_{V}$ versus $\left(m_{P S} / m_{V}\right)^{2}$. Squares: direct lattice calculations of $f_{V}$; blue symbols $f_{V}$ from KSRF $f_{P S}$ and $m_{V}$. The fancy cross is the KSRF result for massless quarks from the physical rho mass and pion decay constant. Results for physical particles are shown in red. (b) The vector meson decay constant $g_{V P P}$ from lattice calculations, as a function of $\left(m_{P S} / m_{V}\right)^{2}$. Symbols are squares, Ref. [19] and [20]; fancy crosses, Ref. [21] and [22]; octagons, Ref. [23]; diamond, Ref. [24] and bursts, Ref. [25]. The line is the KSRF relation with physical values for the rho mass and $f_{\pi}$.

allow scale setting for phenomenology, it is crucial to present intermediate results, i. e. ratios of physical quantities, in addition to final results in units of $\mathrm{GeV}$. Moreover, the most useful ratio for scale setting may be different depending on the BSM model; for example, in dark matter models the mass of the dark matter candidate bound state is a natural choice for scale setting.

One example of a broadly useful and lattice-accessible ratio is the quantity $m_{P S} / f_{P S}$. It sometimes appears as a free parameter in the phenomenological literature, where it is allowed to vary over a large range. (For example, see Fig. 1 of Ref. [14]. This is not $S U(3)$, but that is not important for the point we are about to make.) In QCD, simulations show that this ratio is always smaller than about 5-6. A compilation of lattice data is shown in Fig. 2. The range is even smaller if one wants to be in the chiral regime $\left(m_{P S} / m_{V}\right.$ small). Knowing that simulations can bound the possible ranges of quantities like $m_{P S} / f_{P S}$ can sharpen phenomenological predictions.

Of course, there are many other things to say, but we just conclude with two remarks:

First, there is a market for $S U(3)$ lattice results away from the chiral limit. Your results might enable phenomenologists to sharpen their predictions (and improve them). To enable this, make sure your results are packaged in a broadly useful way: include results for physical quantities even away from the physical point of QCD, and include dimensionless ratios that can be used to set the scale in a variety of ways.

Second, phenomenology makes heavy use of models. "Model" is a heretical word to the lattice community, but that is not so, outside it. It can be useful to present lattice results in a way which allows easy comparison with models, rather than as stand - alone results. The KSRF relations discussed above, which can be obtained from models of vector meson dominance, are a good example. 


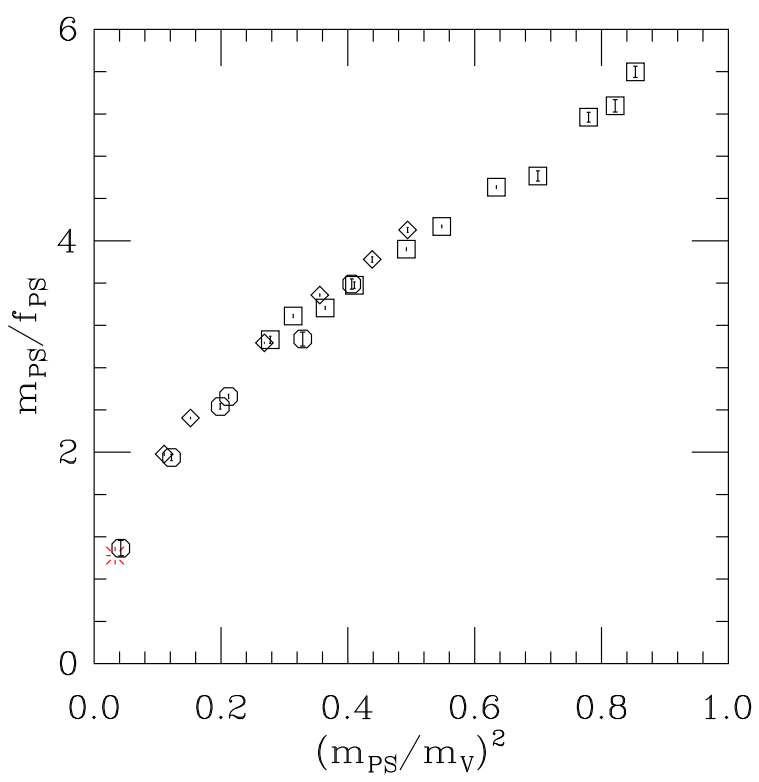

Figure 2: Ratio of pseudoscalar mass to decay constant as a function of $\left(m_{P S} / m_{V}\right)^{2}$. Data are octagons from Ref.[26], diamonds from Ref. [27], and squares from a large statistics follow-on to Ref. [28]. In our convention the physical $f_{\pi}=130 \mathrm{MeV}$, shown as the red burst.

\section{Acknowledgments}

We would like to thank John Bulava, Jim Halverson, and Yuhsin Tsai for conversations and correspondence. This work was supported in part by the U.S. Department of Energy under grant DE-SC0010005. Brookhaven National Laboratory is supported by the U. S. Department of Energy under contract DE-SC0012704.

\section{References}

[1] T. DeGrand, E. T. Neil, paper in preparation.

[2] M. J. Strassler and K. M. Zurek, Phys. Lett. B 651, 374 (2007) doi:10.1016/j.physletb.2007.06.055 [hep-ph/0604261].

[3] M. Pospelov, A. Ritz and M. B. Voloshin, Phys. Lett. B 662, 53 (2008) doi:10.1016/j.physletb.2008.02.052 [arXiv:0711.4866 [hep-ph]].

[4] T. Han, Z. Si, K. M. Zurek and M. J. Strassler, JHEP 0807, 008 (2008) doi:10.1088/1126-6708/2008/07/008 [arXiv:0712.2041 [hep-ph]].

[5] N. Craig, A. Katz, M. Strassler and R. Sundrum, JHEP 1507, 105 (2015) doi:10.1007/JHEP07(2015)105 [arXiv:1501.05310 [hep-ph]].

[6] Z. Chacko, H. S. Goh and R. Harnik, Phys. Rev. Lett. 96, 231802 (2006) doi:10.1103/PhysRevLett.96.231802 [hep-ph/0506256].

[7] H. C. Cheng, S. Jung, E. Salvioni and Y. Tsai, JHEP 1603, 074 (2016) doi:10.1007/JHEP03(2016)074 [arXiv:1512.02647 [hep-ph]]. 
[8] Z. Chacko, D. Curtin, M. Geller and Y. Tsai, arXiv:1803.03263 [hep-ph].

[9] C. J. Morningstar and M. J. Peardon, Phys. Rev. D 60, 034509 (1999) doi:10.1103/PhysRevD.60.034509 [hep-lat/9901004].

[10] Y. Chen et al., Phys. Rev. D 73, 014516 (2006) doi:10.1103/PhysRevD.73.014516 [hep-lat/0510074].

[11] W. J. Lee and D. Weingarten, Phys. Rev. D 61, 014015 (2000) doi:10.1103/PhysRevD.61.014015 [hep-lat/9910008].

[12] G. D. Kribs and E. T. Neil, Int. J. Mod. Phys. A 31, no. 22, 1643004 (2016) doi:10.1142/S0217751X16430041 [arXiv:1604.04627 [hep-ph]].

[13] Y. Hochberg, E. Kuflik, T. Volansky and J. G. Wacker, Phys. Rev. Lett. 113, 171301 (2014) doi:10.1103/PhysRevLett.113.171301 [arXiv:1402.5143 [hep-ph]].

[14] Y. Hochberg, E. Kuflik, H. Murayama, T. Volansky and J. G. Wacker, Phys. Rev. Lett. 115, no. 2 , 021301 (2015) doi:10.1103/PhysRevLett.115.021301 [arXiv:1411.3727 [hep-ph]].

[15] A. Berlin, N. Blinov, S. Gori, P. Schuster and N. Toro, Phys. Rev. D 97, no. 5, 055033 (2018) doi:10.1103/PhysRevD.97.055033 [arXiv:1801.05805 [hep-ph]].

[16] K. Kawarabayashi and M. Suzuki, Phys. Rev. Lett. 16, 255 (1966). doi:10.1103/PhysRevLett.16.255

[17] Riazuddin and Fayyazuddin, Phys. Rev. 147, 1071 (1966). doi:10.1103/PhysRev.147.1071

[18] F. Klingl, N. Kaiser and W. Weise, Z. Phys. A 356, 193 (1996) doi:10.1007/s002180050167 [hep-ph/9607431].

[19] J. Bulava, B. Fahy, B. Hörz, K. J. Juge, C. Morningstar and C. H. Wong, Nucl. Phys. B 910, 842 (2016) doi:10.1016/j.nuclphysb.2016.07.024 [arXiv:1604.05593 [hep-lat]].

[20] J. Bulava, B. Hörz and C. Morningstar, EPJ Web Conf. 175, 05026 (2018) doi:10.1051/epjconf/201817505026 [arXiv:1710.04545 [hep-lat]].

[21] J. J. Dudek et al. [Hadron Spectrum Collaboration], Phys. Rev. D 87, no. 3, 034505 (2013) Erratum: [Phys. Rev. D 90, no. 9, 099902 (2014)] doi:10.1103/PhysRevD.87.034505, 10.1103/PhysRevD.90.099902 [arXiv:1212.0830 [hep-ph]].

[22] D. J. Wilson, R. A. Briceno, J. J. Dudek, R. G. Edwards and C. E. Thomas, Phys. Rev. D 92, no. 9 , 094502 (2015) doi:10.1103/PhysRevD.92.094502 [arXiv:1507.02599 [hep-ph]].

[23] D. Guo, A. Alexandru, R. Molina and M. DÃúring, Phys. Rev. D 94, no. 3, 034501 (2016) doi:10.1103/PhysRevD.94.034501 [arXiv:1605.03993 [hep-lat]].

[24] C. Alexandrou et al., Phys. Rev. D 96, no. 3, 034525 (2017) doi:10.1103/PhysRevD.96.034525 [arXiv:1704.05439 [hep-lat]].

[25] F. Erben, J. Green, D. Mohler and H. Wittig, EPJ Web Conf. 175, 05027 (2018) doi:10.1051/epjconf/201817505027 [arXiv:1710.03529 [hep-lat]].

[26] S. Aoki et al. [PACS-CS Collaboration], Phys. Rev. D 79, 034503 (2009) doi:10.1103/PhysRevD.79.034503 [arXiv:0807.1661 [hep-lat]].

[27] A. Walker-Loud et al., Phys. Rev. D 79, 054502 (2009) doi:10.1103/PhysRevD.79.054502 [arXiv:0806.4549 [hep-lat]].

[28] T. DeGrand and Y. Liu, Phys. Rev. D 94, no. 3, 034506 (2016) Erratum: [Phys. Rev. D 95, no. 1, 019902 (2017)] doi:10.1103/PhysRevD.95.019902, 10.1103/PhysRevD.94.034506 [arXiv:1606.01277 [hep-lat]]. 